\title{
The heterogenic tumor microenvironment of hepatocellular carcinoma and prognostic analysis based on tumor neo-vessels, macrophages and $\alpha$-SMA
}

\author{
MIN FANG ${ }^{1 *}$, JINGPING YUAN ${ }^{2 *}$, MENGYUAN CHEN $^{3}$, ZONGWEN SUN ${ }^{4}$, LULU LIU $^{3}$, \\ GUOPING CHENG ${ }^{5}$, HANGJIE YING ${ }^{1}$, SHIFENG YANG ${ }^{5}$ and MING CHEN $^{1}$ \\ ${ }^{1}$ Department of Radiation Oncology, Zhejiang Cancer Hospital, \\ Zhejiang Key Laboratory of Radiation Oncology \& College of Pharmaceutical Sciences, Zhejiang University, \\ Hangzhou, Zhejiang 310022; ${ }^{2}$ Department of Pathology, Renmin Hospital of Wuhan University, Wuhan, Hubei 430060; \\ ${ }^{3}$ Department of Oncology, The Second Clinical Medical College, Zhejiang Chinese Medical University, \\ Hangzhou, Zhejiang 310053; ${ }^{4}$ Department of Oncology, Jining No. 1 People's Hospital, Jining, \\ Shandong 272011; ${ }^{5}$ Department of Pathology, Zhejiang Cancer Hospital, Hangzhou, Zhejiang 310022, P.R. China
}

Received July 25, 2017; Accepted December 4, 2017

DOI: $10.3892 / \mathrm{ol} .2018 .7946$

\begin{abstract}
The present study was performed to quantify tumor neo-vessels, macrophages and fibroblasts in the tumor microenvironment of hepatocellular carcinoma (HCC) and explore the prognostic factors of HCC. The distribution of tumor neo-vessels, macrophages and fibroblasts was quantified by immunohistochemistry and inverted microscopy with the CRi Nuance multispectral imaging system, and the correlation of these parameters with the clinico-pathological characteristics and overall survival of the patients was analyzed. The number of tumor neo-vessels and macrophages, and density of the fibroblasts, as calculated by the thickness of the tumor stroma in the tumor microenvironment, ranged from 51-429 (median, 218), 110-555 (median, 259) and 35.6-555.5 $\mu \mathrm{m}$ (median, 247.0), respectively. Using the median values as a cutoff, the cases were stratified into high- and low-density groups. Survival analysis demonstrated that the high-density groups regarding macrophages $\left(\chi^{2}=5.249, \mathrm{P}=0.022\right)$ and fibroblasts $\left(\chi^{2}=18.073\right.$, $\mathrm{P}<0.001)$ had a significantly shorter disease-free survival (DFS) than the low-density groups. The high-density tumor neo-vessel group had a shorter DFS with a median of 5 months than the low-density group with a median of 7 months; however, there was
\end{abstract}

Correspondence to: Dr Min Fang or Professor Ming Chen, Department of Radiation Oncology, Zhejiang Cancer Hospital, Zhejiang Key Laboratory of Radiation Oncology \& College of Pharmaceutical Sciences, Zhejiang University, 1 Banshan East Road, Hangzhou, Zhejiang 310022, P.R. China

E-mail: fangmin@zjcc.org.cn

E-mail: chenming@zjcc.org.cn

\section{${ }^{*}$ Contributed equally}

Key words: hepatocellular neoplasms, tumor microenvironment, pathology, metastasis no statistical significance between these two groups $\left(\chi^{2}=1.663\right.$, $\mathrm{P}=0.197)$. Regarding the above three stromal components combined, all of the cases were classified into low-, middle- and high-density groups. Survival analysis demonstrated that the high-density group of stromal components had a shorter DFS than the other two groups with a median of 3 months $\left(\chi^{2}=14.439\right.$, $\mathrm{P}=0.001)$. Multivariate analysis by Cox regression indicated that cirrhosis, metastasis stage, as well as macrophage and fibroblast density were independent prognostic factors. In conclusion, the key elements in the tumor microenvironment, including tumor neo-vessels, macrophages and fibroblasts, were heterogenic in HCC tissues and have significant roles in HCC invasion and metastasis. Stromal components are associated with the prognosis of patients with HCC; the higher the density of stromal components, the poorer the prognosis of patients with HCC.

\section{Introduction}

Hepatocellular carcinoma (HCC) is one of the leading causes of cancer-associated mortality worldwide (1), and the third most lethal cancer type in China, accounting for $>40 \%$ of global HCC-associated deaths (2). Despite recent advances in the diagnosis and treatment of $\mathrm{HCC}$, it remains a highly lethal disease and the survival of most patients remains poor with no significant reduction in the mortality rate over the past decades. Even following radical resection of small HCC (tumor sizes $<3 \mathrm{~cm}$ ), the 5-year postoperative recurrence and metastasis rate remains as high as $43.5 \%$ (3). The majority of $\mathrm{HCC}$-associated deaths results from cancer metastasis. In addition, the heterogeneities of HCC make the problem even worse in clinical oncology (4). It has generally been recognized that cancer invasion, including HCC invasion, is not merely a local problem involving cells in the local tumor microenvironment, but the result of the activity of numerous oncogenic somatic immune cells cancer cells throughout the body (5). With this regard, a fundamental importance lies in changes in the tumor microenvironment (5). The dynamic evolution of cancer cells 
within and at the borders of the tumor microenvironment is crucial in every aspect of tumor progression (5). The role of the tumor microenvironment, including stromal cells and the extracellular matrix (ECM), varies from ECM remodeling to determining the polarity of tissue by linearization of interstitial collagens at tumor invasion front (6), to the recruitment of immune cells as it has been reported that collagen degradation products serve as chemotactic stimuli for monocytes (7), for the acquisition of features defined as 'cancer hallmarks' to generate a physiologically dysfunctional microenvironment. Therefore, rich information hidden in the tumor microenvironment should be systematically investigated to gain a complete understanding of cancer invasion-associated biology.

The ECM has been traditionally regarded as a physical scaffold that holds cells and tissues together, and its remodeling is a common feature of diverse pathological processes, including tissue fibrosis and cancer $(8,9)$. The correlation between tissue fibrosis and cancer has attracted increasing attention from clinicians (10). During cancer invasion, ECM scaffolds undergo considerable structural remodeling, characterized by increased deposition of fibronectin, proteoglycans and collagens, and enhanced matrix cross-linking (11), accompanied with tumor angiogenesis, as well as recruitment and conversion of immune cells (12). The balance of ECM degradation and re-deposition is disrupted, which actively induces epithelial-mesenchymal transition (EMT) of cancer cells $(13,14)$. Consequently, the cancer cells are able to migrate from the ECM and develop metastases through stromal cells and immune responses (15), by participating in signaling transduction between the ECM and cancer cells (16), in which the collagen degradation products recruit monocytes, and immune cells aid collagen reorganization to improve cancer cell penetration (17). In addition, angiogenesis, the formation of tumor neo-vasculature, is essential for any malignant tumor type, including $\mathrm{HCC}$, as tumors must recruit vessels to secure oxygen and nutrient supply, and to remove waste products $(18,19)$. Macrophages also have important roles in cancer cell invasion and metastasis by regulating the immune response of the body to cancer cells and secrete various cytokines (20). A study by Ruffell et al (21) demonstrated that macrophages promote cancer invasion in Hodgkin's lymphoma, while other studies indicated that macrophages inhibit colon cancer invasion (22) or that they have no influence on the prognosis of cancer patients (23).

The present study aimed to assess the density of macrophages, tumor neo-vessels and the expression of $\alpha$-SMA in tumor stroma in order to investigate the heterogeneity of the tumor microenvironment in depth, in an attempt to provide a guide for improving the individual treatment strategy for patients with HCC.

\section{Materials and methods}

Patients and specimens. A total of 3 separate HCC tissue sections $(4 \mu \mathrm{m})$ from 101 patients with HCC were collected from Zhejiang Cancer Hospital (Hangzhou, China) between January 2012 and December 2014. The study protocol was approved by the Institutional Ethics Committee of Zhejiang Cancer Hospital (Hangzhou, China) and the patients provided written informed consent regarding the use of their tissues. Major clinicopathological features of these patients are listed in Table I. All of the cases underwent curative radical surgery without neo-adjuvant radiotherapy or chemotherapy. Tumor staging was based on the tumor-nodes-metastasis (TNM) classification system of the American Joint Committee on Cancer staging criteria (version 7) (24). The median follow-up was 41 months. Disease-free survival (DFS) was defined as the interval from the date of surgery to recurrence, and if no recurrence was identified, patients were censored on the date of death or the last follow-up. Evidence of disease recurrence was based on the following criteria: The concentration of AFP (>200 ng/ml), local recurrence identified on ultrasonography, computed tomography (CT) or magnetic resonance imaging; peritoneal dissemination on ultrasonography or CT scan with positive peritoneal cytology; lung metastasis on chest radiography; and bone metastasis on radiography or bone scan on emission computed tomography.

Immunohistochemical staining. Immunohistochemical staining of macrophages marked by CD68, tumor neo-vessels marked by CD105 and $\alpha$-SMA were performed using the streptavidin-biotin peroxidase complex method. In brief, tissue slides were first deparaffinized in xylene, ethanol and water, and then the slides were treated with $0.01 \mathrm{M}$ citrate buffer (pH 6.0) and heated in a microwave oven at $98^{\circ} \mathrm{C}$ for $10 \mathrm{~min}$. For staining, endogenous peroxidase activity was blocked by immersing samples in $3 \% \mathrm{H}_{2} \mathrm{O}_{2}$ in methanol for $10 \mathrm{~min}$ at $37^{\circ} \mathrm{C}$ to prevent any nonspecific binding. After blocking with $2 \%$ bovine serum albumin (Shanghai Ruji Biotechnology, Shanghai, China), the slides were incubated with primary antibodies to CD68 (cat. no. MA1-38069; dilution, 1/300; Affinity BioReagents, Golden, CO, USA), CD105 (ab-169545; dilution, 1/300; Abcam, Cambridge, MA, USA) and $\alpha$-SMA (ab-5694; dilution, 1/300; Abcam) for $90 \mathrm{~min}$ at $37^{\circ} \mathrm{C}$, and then incubated with the corresponding secondary antibody for $15 \mathrm{~min}$ at $37^{\circ} \mathrm{C}$, and finally incubated with peroxidase-labeled streptavidin (cat. no., 091014395C; dilution, 1:250; Maixin Biotechnology, Fuzhou, China) for $15 \mathrm{~min}$. The reaction products were visualized with diaminobenzidine (DAKO, Glostrup, Denmark). All slides were counterstained with hematoxylin for $2 \mathrm{~min}$ at room temperature. As a negative control, the primary antibody was replaced with Tris-buffered saline on sections.

Quantification of immunohistochemical staining. The slides were examined under an Olympus BX51 microscope equipped with an Olympus DP72 camera (Olympus Optical Co., Ltd., Tokyo, Japan) and the CRi Nuance multispectral imaging system (Cambridge Research \& Instrumentation, Inc., Woburn, MA, USA). Positive staining was indicated by brownish granules. A spectral cube for each image, which contains the complete spectral information at $10 \mathrm{~nm}$ wavelength intervals from 420 to $720 \mathrm{~nm}$, was collected using the CRi Nuance multispectral imaging system. For each cube identical settings were used to avoid selection bias. Positive signal unmixing was performed using the software package within the Nuance system as previously described (25). Then, after obtaining the images of signal unmixing, the infiltrating macrophages marked by CD68 and tumor neo-vessels marked by CD105 were counted in five high-power fields selected at the tumor site, and the mean cells counts were documented. The thickness of infiltrating stroma marked by $\alpha$-SMA was measured to 
Table I. Major demographic and clinicopathological characteristics of hepatocellular carcinoma cases $(n=101)$.

\begin{tabular}{|c|c|}
\hline Item & Value \\
\hline Age (years) & $51(19-75)$ \\
\hline \multicolumn{2}{|l|}{ Sex } \\
\hline Male & $90(89.1)$ \\
\hline Female & $11(10.9)$ \\
\hline \multicolumn{2}{|l|}{ Tumor size (cm) } \\
\hline$\leq 5$ & $26(25.7)$ \\
\hline$>5$ & $75(74.3)$ \\
\hline \multicolumn{2}{|l|}{ Tumor number } \\
\hline Single & $86(85.1)$ \\
\hline Multiple & 15 (14.9) \\
\hline \multicolumn{2}{|l|}{$\operatorname{AFP}(n g / m l)$} \\
\hline$\leq 200$ & $54(53.5)$ \\
\hline$>200$ & $47(46.5)$ \\
\hline \multicolumn{2}{|l|}{ Liver cirrhosis } \\
\hline No & $58(57.4)$ \\
\hline Yes & $43(42.6)$ \\
\hline \multicolumn{2}{|l|}{ Venous infiltration } \\
\hline No & $53(52.5)$ \\
\hline Yes & $48(47.5)$ \\
\hline \multicolumn{2}{|l|}{ TNM stage } \\
\hline Early (Stage I, II) & $47(46.5)$ \\
\hline Advanced (Stage III, IV) & $54(53.5)$ \\
\hline \multicolumn{2}{|l|}{ Tumor recurrence } \\
\hline No & 37 (36.6) \\
\hline Yes & $64(63.4)$ \\
\hline \multicolumn{2}{|l|}{ Distant metastasis } \\
\hline No & $46(45.5)$ \\
\hline Yes & $55(54.5)$ \\
\hline DFS (Median, range) & $5.0(0-54.0)$ \\
\hline
\end{tabular}

Values are expressed as median (range) or $\mathrm{n}(\%)$. TNM, tumor-nodes-metastasis; AFP, $\alpha$-fetoprotein; DFS, disease-free survival.

evaluate the depth between tumor nests. For determining the density of infiltrating macrophages, neo-vessels density and thickness of the stroma, the cutoff value to classify subgroups was 270,215 and $238.6 \mu \mathrm{m}$ respectively (26), according to the mean value.

Statistical analysis. Statistical analyses were performed with SPSS software version 21.0 (IBM Corp., Armonk, NY, USA). For categorical data, $\chi^{2}$ test was performed. The Kaplan-Meier method was used to estimate survival curves for DFS and the log-rank test was used to assess the difference in survival and recurrence rates between subgroups. The Cox regression model was used to perform univariate and multivariate analyses. Logistic regression was used to assess the influence of binary factors. A two-sided $\mathrm{P}<0.05$ was considered to indicate a statistically significant difference.

\section{Results}

Major clinicopathological features of 101 HCC cases. Among the $101 \mathrm{HCC}$ cases included in this study, 90 (89.1\%) were males and $11(10.9 \%)$ were females, ranging in age from 19 to 75 years (median age, 51 years). The major demographic and clinicopathological characteristics are presented in Table I. Immunohistochemical analysis was performed on all HCC tissue sections and the corresponding images of signal unmixing for tumor neo-vessels and macrophages were obtained with the CRi Nuance multispectral imaging system (Fig. 1).

Association between tumor neo-vessels, macrophages, $\alpha$-SMA and clinicopathological features. CD105, CD68 and $\alpha$-SMA staining was mainly located in the cytoplasm or on the cell membrane. Tumor neo-vessels (stained by CD105) and macrophages (stained by CD68) were distributed in tumor nests as well as in tumor stroma. $\alpha$-SMA staining was mainly located in the tumor stroma. The majority of the cancer cells were negative for $\alpha$-SMA staining, although sporadic positive staining on these cells was also observed. However, all above components, including tumor neo-vessels, macrophages and $\alpha$-SMA, were heterogeneously expressed in the tumor microenvironment (Fig. 2). In certain cases, only few tumor neo-vessels and macrophages were present compared with other cases with a rich distribution in tumor tissues. The amount of $\alpha$-SMA distributed in the stroma was also different among the patients.

The quantitative data of tumor neo-vessels, macrophage density and stromal thickness are presented in Table II. For infiltrating macrophage density, neo-vessel density and thickness of the stroma, the cutoff value to classify subgroups was 270,215 and $238.6 \mu \mathrm{m}$ respectively. In the high and low tumor neo-vessel subgroups, 24 (52.2\%) and $16(36.4 \%)$ of cases, respectively, had a high macrophage density. In the high and low $\alpha$-SMA subgroups, 29 (58.0\%) and 19 (40.4\%) of cases, respectively, had a high tumor neo-vessel density, and $23(48.9 \%)$ and 17 (39.5\%) of cases, respectively, had a high macrophage density. Of note, the tumor neo-vessel, macrophage and $\alpha$-SMA densities were not significantly correlated with any of the clinicopathological features, as summarized in Table II, which indicated that these key components of the tumor microenvironment were independent from clinical features including tumor size, tumor number, TNM stage and venous infiltration.

Survival analysis. For the 101 HCC cases, the median DFS was 5.0 months (range, 22 days-54.0 months). As expected, several clinical factors were associated with the DFS of HCC patients, including tumor size, venous infiltration and tumor recurrence $(\mathrm{P}<0.05$ for all). Furthermore, tumor macrophage and $\alpha$-SMA densities were negatively associated with DFS ( $\mathrm{P}=0.022$ and $\mathrm{P}<0.001$, respectively). In addition, the combined features based on the above three key components in the tumor microenvironment were explored to reveal the association between key stromal factors with HCC prognosis. According to the expression levels of the three key stromal components, patients were divided into three subgroups according to the density of tumor neo-vessels, macrophages and $\alpha$-SMA: Group I, all 


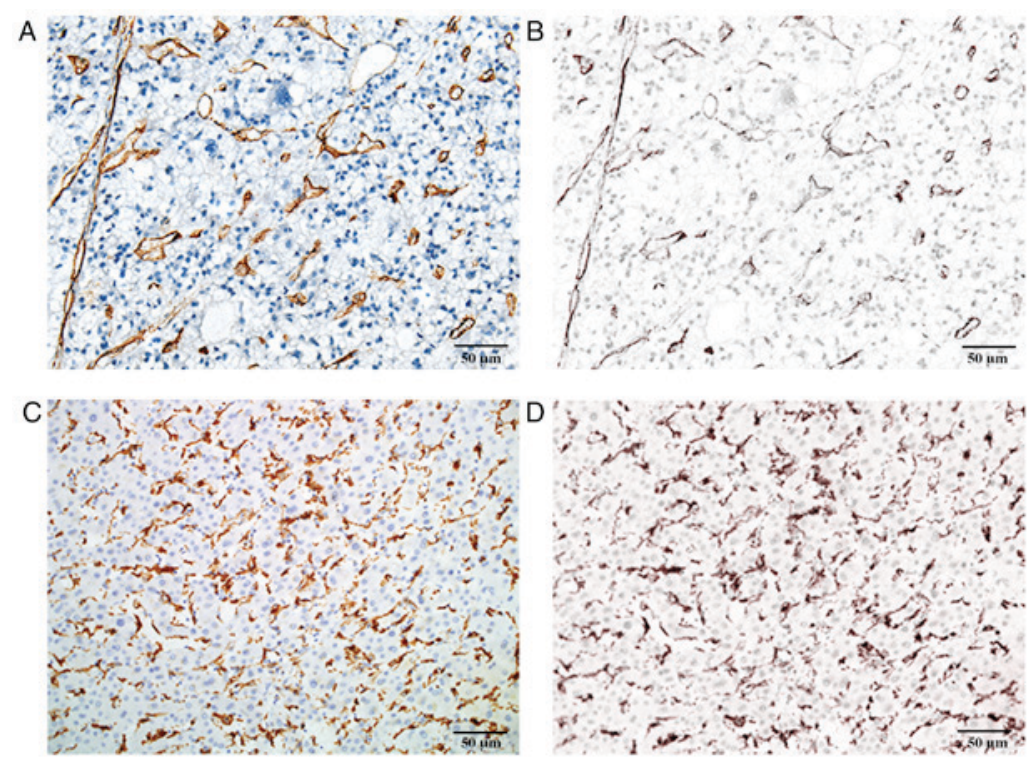

Figure 1. Immunohistological analysis of hepatocellular carcinoma tissue sections. Representative immunohistochemical images of (A and B) tumor neo-vessels stained for CD105 and of (C and D) macrophages stained for CD68. B and D display the corresponding images following signal unmixing with the CRi Nuance multispectral imaging system (scale bar, $50 \mu \mathrm{m}$ ).
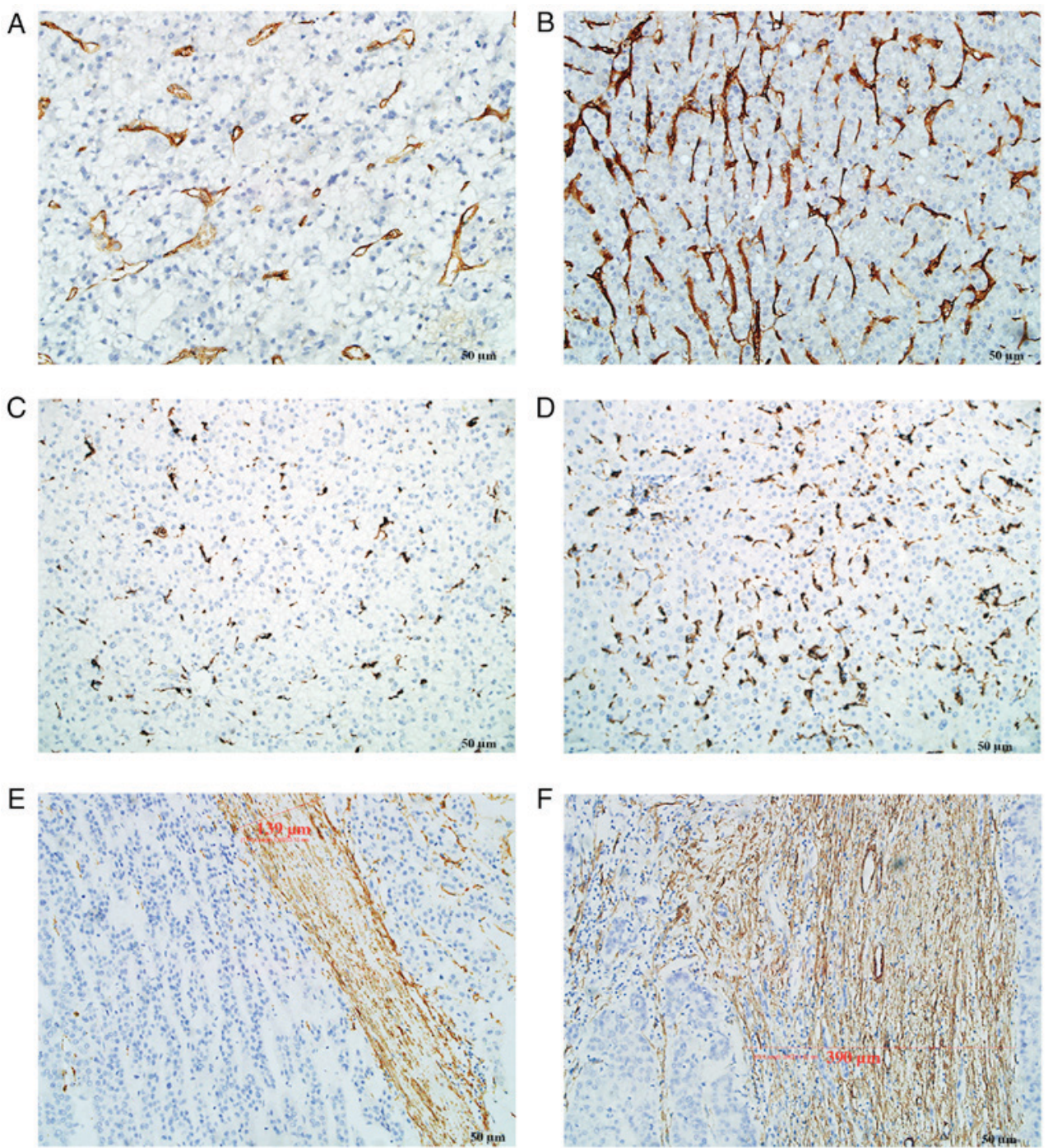

Figure 2. Heterogeneous density of stromal components in the hepatocellular carcinoma microenvironment. (A and B) Tumor neo-vessels in HCC with (A) scattered and discrete distribution and (B) high density. (C and D) Macrophages in HCC tissue with (C) low and (D) high density. (E and F) Fibroblasts in HCC tissues with (E) low and (F) high density as determined by immunohistochemical detection of $\alpha$-smooth muscle actin (scale bar, $50 \mu \mathrm{m}$ ). 


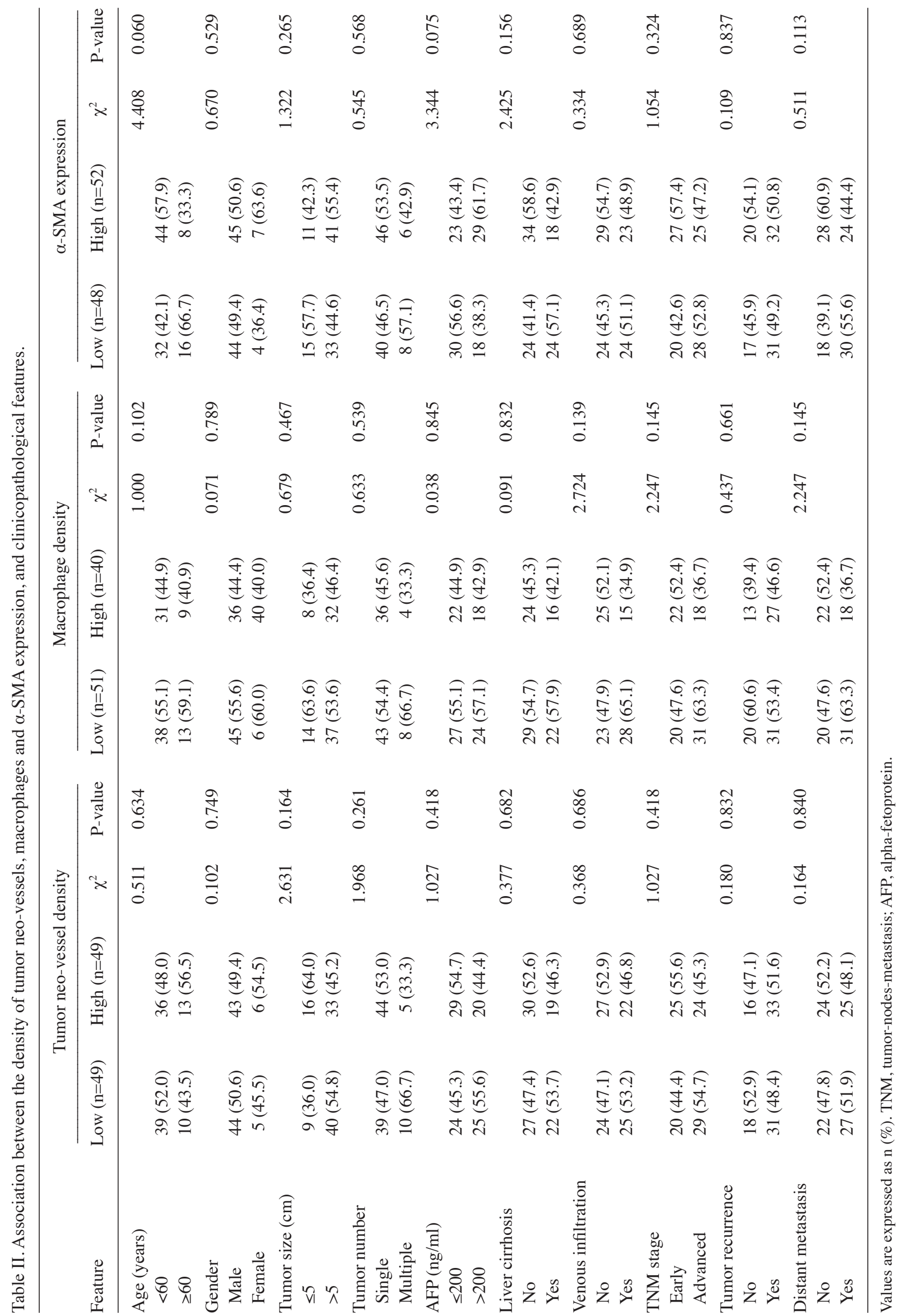



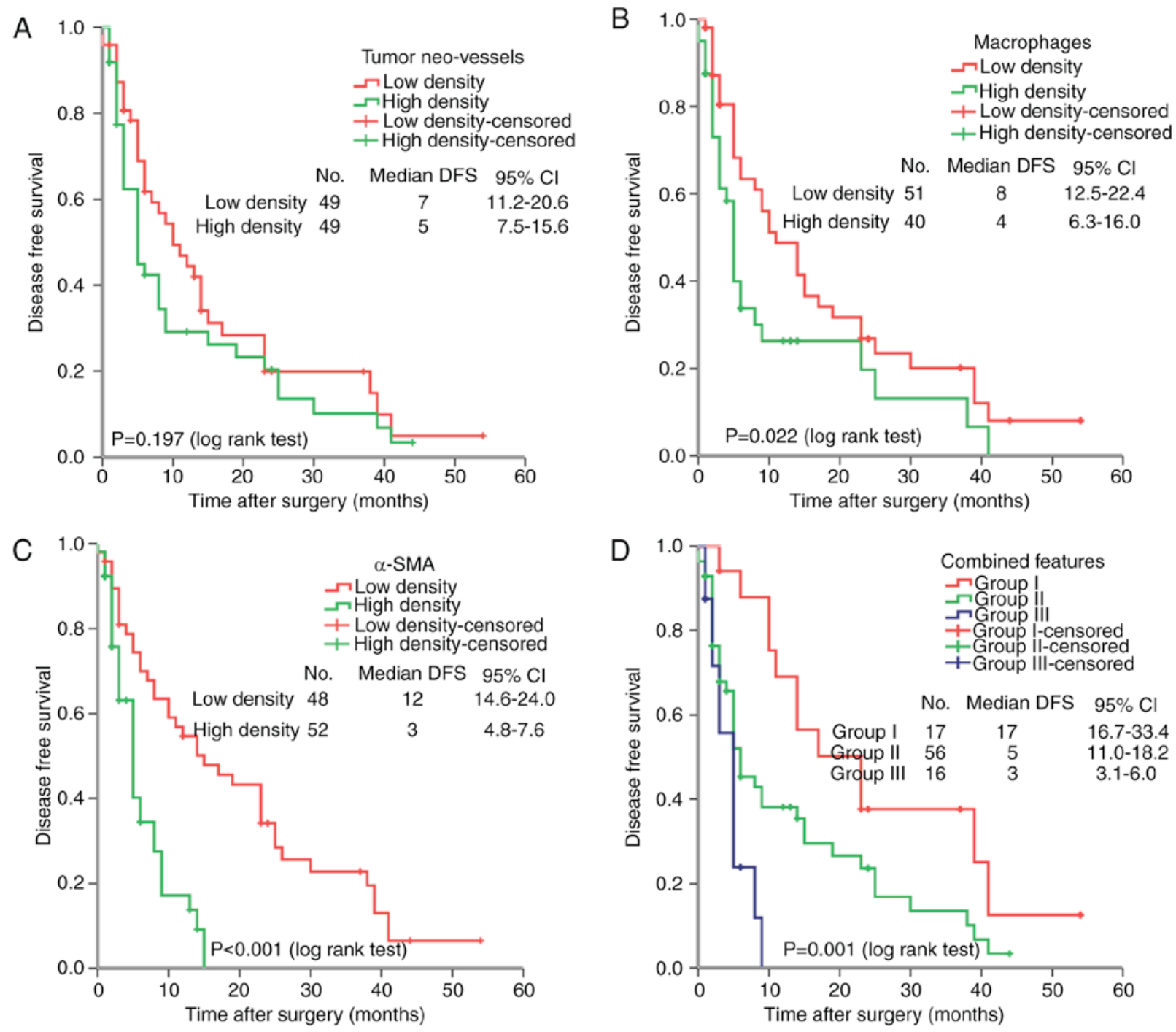

Figure 3. Cumulative DFS of patients with HCC. (A) Tumor neo-vessels were not associated with DFS of patients with HCC. (B and C) Patients in the high-density (B) macrophage and (C) $\alpha$-SMA expression groups had a higher risk of death. (D) Combined features accurately indicated DFS of patients with HCC. HCC, hepatocellular carcinoma; DFS, disease-free survival; CI, confidence interval; SMA, smooth muscle actin. Group I, all three components were expressed at a low level; group II, 1 or 2 components were expressed at a high level; group III, 3 components were expressed at a high level.

three components were expressed at a low level; group II, 1 or 2 components were expressed at a high level; group III, three components were expressed at a high level. Combined analysis indicated that patients in group III had a worse DFS than those in groups I and II (P=0.001; Fig. 3D).

Multivariate analysis. In univariate analysis, traditional clinicopathological features (including tumor size, venous infiltration and recurrence status), macrophage density and $\alpha$-SMA were associated with DFS. In addition, analysis of the combined key stromal components of tumor neo-vessels, macrophages and $\alpha$-SMA indicated that the mortality risk in combined group III was significantly increased $(\mathrm{P}=0.001)$.

As presented in Table III, in the multivariate analysis Model 1, all factors were integrated into a multivariate Cox proportional hazards analysis. Liver cirrhosis status, tumor recurrence status, macrophage density and $\alpha$-SMA density were independent prognostic factors for DFS $(\mathrm{P}<0.05$ for all). In Model 2 , factors exhibiting significance according to a univariate analysis were integrated into a multivariate Cox proportional hazards analysis in order to avoid multicollinearity among those variables. Tumor recurrence status, macrophage density and $\alpha$-SMA density were independent prognostic factors for DFS $(\mathrm{P}<0.05$ for all).

\section{Discussion}

It has generally been recognized that cancer invasion, including HCC invasion, is not merely a local problem, but a multifactor and multistep continuum, with a variety of molecular dysfunction and cell signaling dysregulation. The genetic or epigenetic changes of cancer cells are the initial factors' of carcinogenesis, and the responses of stromal cells in the tumor microenvironment may 'promote' or 'regulate' cancer invasion and metastasis, which ultimately results in an altered tumor microenvironment favoring cancer invasion and progression (27). The temporal-spatial evolution of the microenvironment and the interplay between cancer cells and their microenvironment are critical in every aspect of tumor development, including cancer cell dormancy, proliferation, invasion 
Table III. Multivariate analyses of factors associated with DFS.

\begin{tabular}{|c|c|c|c|c|c|c|}
\hline \multirow[b]{2}{*}{ Clinicopathological factor } & \multicolumn{3}{|c|}{ Univariate analysis } & \multicolumn{3}{|c|}{ Multivariate analysis } \\
\hline & P-value & HR & $95 \% \mathrm{CI}$ & P-value & HR & $95 \% \mathrm{CI}$ \\
\hline Age (<60 vs. $\geq 60$ years $)$ & 0.284 & 1.014 & $0.988-1.040$ & & & \\
\hline Sex (female vs. male) & 0.381 & 0.588 & $0.180-1.926$ & & & \\
\hline Tumor size ( $\leq 5$ vs. $>5$ ) & 0.979 & 0.989 & $0.442-2.216$ & 0.181 & 1.552 & $0.815-2.956$ \\
\hline Tumor number (single vs. multiple) & 0.402 & 0.558 & $0.142-2.188$ & & & \\
\hline $\operatorname{AFP}(\leq 200$ vs. $>200)$ & 0.088 & 1.742 & $0.921-3.293$ & & & \\
\hline Liver cirrhosis (no vs. yes) & 0.020 & 0.514 & $0.294-0.899$ & & & \\
\hline Venous infiltration (no vs. yes) & 0.141 & 0.277 & $0.050-1.530$ & 0.696 & 1.114 & $0.649-1.910$ \\
\hline TNM stage (early vs. advanced) ${ }^{\mathrm{a}}$ & 0.098 & 4.955 & $0.745-32.937$ & & & \\
\hline Tumor recurrence (no vs. yes) & $<0.001$ & 28.416 & $7.979-101.205$ & $<0.001$ & 17.035 & $5.644-51.415$ \\
\hline Distant metastasis (no vs. yes) & 0.411 & 0.755 & $0.386-1.476$ & & & \\
\hline Tumor neo-vessel density (low vs. high) & 0.794 & 1.000 & $0.997-1.002$ & & & \\
\hline Macrophage density (low vs. high) & 0.008 & 1.004 & $1.001-1.007$ & 0.025 & 1.870 & $1.082-3.233$ \\
\hline$\alpha$-SMA expression (low vs. high) & 0.005 & 1.004 & $1.001-1.007$ & & & \\
\hline
\end{tabular}

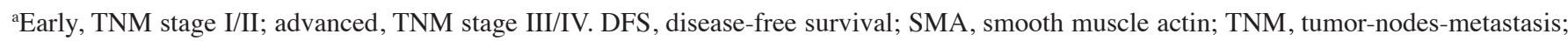
HR, hazard ratio; CI, confidence interval.

and migration (5). Currently, the importance of the tumor microenvironment has been increasingly appreciated, from the physical structures to chemical components, particularly the role of macrophages and tumor neo-vessels and the structure of tumor stroma. Inflammation and tumor angiogenesis are two indispensable factors for cancer invasion (28). The tumor mass relies on blood vessels to gain nutrients and dispose of waste in order to keep growing (29). Typical features of tumor neo-vessels, including irregular shape, disordered structure, high permeability and immaturity contribute to the spreading of tumor cells to distant organs (30). However, the present study demonstrated that tumor neo-vessels are not significantly associated with the prognosis of HCC patients. It is therefore suggested that other key components in the tumor microenvironment, e.g. macrophages, have presumed the significant role of tumor neo-vessels in the process of cancer invasion and metastasis. Tumor-associated macrophages have crucial roles in tumor angiogenesis, including prompting the production of vascular endothelial cells, boosting vessel sprouting and accelerating cancer invasion and metastasis (31). The results of the present study indicated that macrophages indeed have a profound impact on HCC progression and are independent prognostic factors for HCC. In addition, macrophages synthesize other stromal matrix components, including hyaluronic acid, matrix metalloproteinase and fibroblast-activated proteins, all of which may contribute to cancer invasion and metastasis (32). In the present study, it was also confirmed that the density of tumor stroma marked by $\alpha$-SMA was highly associated with HCC prognosis and was an independent prognostic factor for $\mathrm{HCC}$ patients. Numerous other studies have reported that fibroblasts promote lymph node metastasis $(33,34)$. It has been suggested that the biological characteristics of fibroblasts in normal tissues are exceptionally distinguished from those in cancer tissues (35). The majority of fibroblasts are located at the invasion frontier, the interface between tumor mass and surrounding stroma, or near the tumor neo-vessels in the tumor microenvironment, regulating the growth and invasion of cancer cells by synthetizing and remodeling ECM, participating in the process of tumor angiogenesis and downregulating the of the anti-tumor immune response (36). In the present study, increased of fibroblast density indicated thicker stroma and worse prognosis. It was previously reported that the stiffness of the stroma is closely associated with the risk of carcinogenesis (37) and fibrosis is relevant to poor prognosis of cancer patients (38). Tumors are normally characterized as lumps of great toughness, which prompted oncologists to investigate the concept that tumor progression may be eased by decreasing the toughness of tissues (39). Others have confirmed that only a slight change in the toughness of stroma or in mechanical signals affects the biological behavior of cells (40). Thus, the significance of research into the tumor microenvironment is particularly prominent.

In conclusion, key components in the tumor microenvironment may interact with cancer cells, and together accelerate the cancer invasion and metastasis. The present study implied that tumor-associated macrophages and the expression of $\alpha$-SMA are intimately linked with the prognosis of HCC patients. However, further research using larger amounts of samples is still required to confirm and interpret relevant mechanisms in more depth.

\section{Acknowledgements}

This study was supported by the Zhejiang Medical and Health Science and Technology Project (grant no. 2016KYA048), the Zhejiang Natural Science Foundation (grant no. LQ17H180003), and the Postdoctoral Program of Zhejiang Province and the Chinese Postdoctoral Fund (grant no. 520000-X91601). 


\section{References}

1. Siegel RL, Miller KD and Jemal A: Cancer statistics, 2017. CA Cancer J Clin 67: 7-30, 2017.

2. Chen W, Zheng R, Baade PD, Zhang S, Zeng H, Bray F, Jemal A, Yu XQ and He J: Cancer statistics in China, 2015. CA Cancer J Clin 66: 115-132, 2016

3. Tang ZY, Ye SL, Liu YK, Qin LX, Sun HC, Ye QH, Wang L, Zhou J, Qiu SJ, Li Y, et al: A decade's studies on metastasis of hepatocellular carcinoma. J Cancer Res Clin Oncol 130: 187-196, 2004.

4. Marusyk A and Polyak K: Tumor heterogeneity: Causes and consequences. Biochim Biophys Acta 1805: 105-117, 2010.

5. Hanahan D and Weinberg RA: Hallmarks of cancer: The next generation. Cell 144: 646-674, 2011.

6. Paszek MJ, Zahir N, Johnson KR, Lakins JN, Rozenberg GI, Gefen A, Reinhart-King CA, Margulies SS, Dembo M, Boettiger $\mathrm{D}$, et al: Tensional homeostasis and the malignant phenotype. Cancer Cell 8: 241-254, 2005.

7. Weathington NM, van Houwelingen AH, Noerager BD, Jackson PL, Kraneveld AD, Galin FS, Folkerts G, Nijkamp FP and Blalock JE: A novel peptide CXCR ligand derived from extracellular matrix degradation during airway inflammation. Nat Med 12: 317-323, 2006.

8. Schäfer M and Werner S: Cancer as an overhealing wound: An old hypothesis revisited. Nat Rev Mol Cell Biol 9: 628-638, 2008.

9. Araya J and Nishimura SL: Fibrogenic reactions in lung disease. Annu Rev Pathol 5: 77-98, 2010.

10. Martin LJ and Boyd NF: Mammographic density. Potential mechanisms of breast cancer risk associated with mammographic density: Hypotheses based on epidemiological evidence. Breast Cancer Res 10: 201, 2008.

11. Egeblad M, Rasch MG and Weaver VM: Dynamic interplay between the collagen scaffold and tumor evolution. Curr Opin Cell Biol 22: 697-706, 2010.

12. Polyak K, Haviv I and Campbell IG: Co-evolution of tumor cells and their microenvironment. Trends Genet 25: 30-38, 2009.

13. Friedl P, Locker J, Sahai E and Segall JE: Classifying collective cancer cell invasion. Nat Cell Biol 14: 777-783, 2012.

14. Barker HE, Cox TR and Erler JT: The rationale for targeting the LOX family in cancer. Nat Rev Cancer 12: 540-552, 2012.

15. Nerenberg PS, Salsas-Escat R and Stultz CM: Collagen-a necessary accomplice in the metastatic process. Cancer Genomics Proteomics 4: 319-328, 2007.

16. Torzilli PA, Bourne JW, Cigler T and Vincent CT: A new paradigm for mechanobiological mechanisms in tumor metastasis. Semin Cancer Biol 22: 385-395, 2012.

17. Fang M, Yuan J, Peng C, and Li Y: Collagen as a double-edged sword in tumor progression. Tumour Biol 35: 2871-2882, 2014.

18. Kerbel RS: Tumor angiogenesis. N Engl J Med 358: 2039-2049, 2008.

19. Kappler M, Taubert $H$ and Eckert AW: Oxygen sensing, homeostasis, and disease. N Engl J Med 365: 1845-1846, 2011.

20. Giraldo NA, Becht E, Remark R, Damotte D, Sautès-Fridman C and Fridman WH: The immune contexture of primary and metastatic human tumours. Curr Opin Immunol 27: 8-15, 2014.

21. Ruffell B, Affara NI and Coussens LM: Differential macrophage programming in the tumor microenvironment. Trends Immunol 33: 119-126, 2012.

22. Zhou Q, Peng RQ, Wu XJ, Xia Q, Hou JH, Ding Y, Zhou QM, Zhang X, Pang ZZ and Wan DS: The density of macrophages in the invasive front is inversely correlated to liver metastasis in colon cancer. J Transl Med 8: 13, 2010.

23. Azambuja D, Natkunam Y, Biasoli I, Lossos IS, Anderson MW, Morais JC and Spector N: Lack of association of tumor-associated macrophages with clinical outcome in patients with classical Hodgkin's lymphoma. Ann Oncol 23: 736-742, 2012.
24. Edge SB: AJCC Cancer stagin Manual. 7th edition. New York, NY: Springer. Liver, pp201-210, 2007.

25. Taylor CR and Levenson RM: Quantification of immunohistochemistry-issues concerning methods, utility and semiquantitative assessment II. Histopathology 49: 411-424, 2006.

26. Olsson PO, Kalamajski S, Maccarana M, Oldberg Å and Rubin K Fibromodulin deficiency reduces collagen structural network but not glycosaminoglycan content in a syngeneic model of colon carcinoma. PLoS One 12: e0182973, 2017.

27. Hoon DS, Kitago M, Kim J, Mori T, Piris A, Szyfelbein K, Mihm MC Jr, Nathanson SD, Padera TP, Chambers AF, et al: Molecular mechanisms of metastasis. Cancer Metastasis Rev 25: 203-220, 2006

28. Squadrito ML and De Palma M: Macrophage regulation of tumor angiogenesis: Implications for cancer therapy. Mol Aspects Med 32: 123-145, 2011.

29. Semenza GL: Oxygen sensing, homeostasis, and disease. N Engl J Med 365: 537-547, 2011.

30. Muz B, de la Puente P, Azab F and Azab AK: The role of hypoxia in cancer progression, angiogenesis, metastasis, and resistance to therapy. Hypoxia (Auckl) 3: 83-92, 2015.

31. Mantovani A and Allavena P: The interaction of anticancer therapies with tumor-associated macrophages. J Exp Med 212: 435-445, 2015.

32. Erler JT, Bennewith KL, Cox TR, Lang G, Bird D, Koong A, Le QT and Giaccia AJ: Hypoxia-induced lysyl oxidase is a critical mediator of bone marrow cell recruitment to form the premetastatic niche. Cancer Cell 15: 35-44, 2009.

33. Liang P, Hong JW, Ubukata H, Liu G, Katano M, Motohashi G, Kasuga T, Watanabe Y, Nakada I and Tabuchi T: Myofibroblasts correlate with lymphatic microvessel density and lymph node metastasis in early-stage invasive colorectal carcinoma. Anticancer Res 25: 2705-2712, 2005.

34. Kellermann MG, Sobral LM, da Silva SD, Zecchin KG, Graner E, Lopes MA, Kowalski LP and Coletta RD: Mutual paracrine effects of oral squamous cell carcinoma cells and normal oral fibroblasts: Induction of fibroblast to myofibroblast transdifferentiation and modulation of tumor cell proliferation. Oral Oncol 44: 509-517, 2008.

35. Castells M, Thibault B, Delord JP and Couderc B: Implication of tumor microenvironment in chemoresistance: Tumor-associated stromal cells protect tumor cells from cell death. Int J Mol Sci 13: 9545-9571, 2012.

36. Cirri P and Chiarugi P: Cancer-associated-fibroblasts and tumour cells: A diabolic liaison driving cancer progression. Cancer Metastasis Rev 31: 195-208, 2012.

37. Provenzano PP, Inman DR, Eliceiri KW, Knittel JG, Yan L, Rueden CT, White JG and Keely PJ: Collagen density promotes mammary tumor initiation and progression. BMC Med 6: 11, 2008.

38. Baba Y, Iyama K, Ikeda K, Ishikawa S, Hayashi N, Miyanari N, Sado Y, Ninomiya $\mathrm{Y}$ and Baba $\mathrm{H}$ : The expression of type IV collagen alpha6 chain is related to the prognosis in patients with esophageal squamous cell carcinoma. Ann Surg Oncol 15: 555-565, 2008.

39. Susic D: Cross-link breakers as a new therapeutic approach to cardiovascular disease. Biochem Soc Trans 35: 853-856, 2007.

40. McDaniel SM, Rumer KK, Biroc SL, Metz RP, Singh M, Porter W and Schedin P: Remodeling of the mammary microenvironment after lactation promotes breast tumor cell metastasis. Am J Pathol 168: 608-620, 2006.

This work is licensed under a Creative Commons Attribution-NonCommercial-NoDerivatives 4.0 International (CC BY-NC-ND 4.0) License. 